\title{
Cavity flow induced by a fluctuating acceleration field
}

\author{
J. Ross Thomson, ${ }^{\text {a) }}$ Jaume Casademunt, ${ }^{\text {b) }}$ and Jorge Viñals \\ Supercomputer Computations Research Institute, Florida State University, Tallahassee, Florida 32306-4052
}

(Received 11 April 1994; accepted 14 October 1994)

Buoyancy driven convection induced by a fluctuating acceleration field is studied in a two dimensional square cavity. This is a simplified model of, for example, fluid flow in a directional solidification cell subject to external accelerations, such as those encountered in a typical microgravity environment ( $g$-jitter). The effect of both deterministic and stochastic acceleration modulations normal to the initial density gradient are considered. In the latter case, the acceleration field is modeled by narrow band noise defined by a characteristic frequency $\Omega$, a correlation time $\tau$, and an intensity $G^{2}$. If the fluid is quiescent at $t=0$ when the acceleration field is initiated, the ensemble average of the vorticity at the center of the cavity remains zero for all times. The mean squared vorticity $\left\langle\xi^{2}\right\rangle$, however, is seen to exhibit two distinct regimes: For $t \ll \tau,\left\langle\xi^{2}\right\rangle$ oscillates in time with frequency $\Omega$. For $t \gg \tau,\left\langle\xi^{2}\right\rangle$ grows linearly in time with an amplitude equal to $\mathrm{R}^{2} \operatorname{Pr} /(1+(\Omega \tau))^{2}$, where $\mathrm{R}$ is a new dimensionless number which reduces to the Rayleigh number in the case of a constant gravity, and $\operatorname{Pr}$ is Prandtl number. At yet later times, viscous dissipation at the walls of the cavity leads to saturation, with $\left\langle\xi^{2}\right\rangle_{\text {sat }}=\left\{(\operatorname{Pr} \tau+1) R^{2} /\left[(\operatorname{Pr} \tau+1)^{2}+\Omega^{2} \tau^{2}\right]\right\}$. (C) 1995 American Institute of Physics.

\section{INTRODUCTION}

Frequent access to a microgravity environment has permitted numerous fluid and materials science experiments designed to take advantage of near weightlessness. In some cases, the aim is to study new phenomena otherwise masked by the Earth's gravitational field, while in others, it is hoped to gain the necessary knowledge to implement materials processing methods thought to be adversely affected by the Earth's gravitational field. The best examples of the latter case are the growth of semiconductor or protein crystals. However, recent systematic characterizations of the effective acceleration environment aboard spacecraft have shown that the residual acceleration field is not constant but comprises a broad frequency spectrum. At low frequencies (less than $10^{-2} \mathrm{~Hz}$ ), the Fourier amplitudes of the residual field are approximately $10^{-6} g_{E}$ (where $g_{E}$ is the intensity of the gravitational field on the Earth's surface). At frequencies of about $1 \mathrm{~Hz}$, however, the amplitude is of the order of $10^{-4} g_{E}-10^{-3} g_{E}$, increasing roughly linearly with frequency for higher frequencies. ${ }^{1}$ The effect that such large amplitude and high frequency components of the residual acceleration field ( $g$-jitter) can have on experiments conducted in space is largely unknown.

The residual acceleration field can be decomposed for practical purposes into two contributions, a quasi-steady or systematic component $\mathbf{g}_{s}(t)$ (either constant or changing very slowly in time), and a fluctuating contribution $\mathrm{g}(t)$ ( $\mathrm{g}$ jitter). The fluctuating contribution $\mathbf{g}$ is thought to be of inherently local and statistical nature. Its origins are both processes that take place outside the spacecraft (e.g., thruster firings) and, more importantly, internal motion of mechanical parts, crew activities and other experiments. Characteristic frequencies of $g$-jitter are $1 \mathrm{~Hz}$ or higher and their amplitude

\footnotetext{
a)e-mail: thomson@scri.fsu.edu, tel: (904)-644-2434, fax: (904)-644-0098

b) Present address: Departament d'Estructura i Constituents de la Materia, Universitat de Barcelona, 08028 Barcelona, Spain.
}

is two or more orders of magnitude larger than the quasisteady component. For the purposes of the analysis presented in this paper, we consider only the fluctuating contribution, g, and take its average to be zero. Although it would not contribute significantly to the difficulty of the analysis, the addition of a constant component would provide little additional insight.

In this paper, we study the onset of natural convection in a fluid that is enclosed in a laterally heated, two dimensional square cavity, and is subjected to a stochastic, time dependent acceleration field normal to the imposed temperature gradient. We expect our analysis to capture many of the essential characteristics of buoyancy induced convection in the type of fluctuating acceleration field measured aboard spacecraft.

Classical studies of natural convection under a constant acceleration field in the two dimensional configuration studied here are divided in two classes according to the predominant mode of heat transfer across the cell: conduction dominated and convection dominated transfer. ${ }^{2-4}$ Numerical studies have also been performed for parameters between the two extremes of convection dominance and conduction dominance, since this intermediate regime cannot be treated analytically. ${ }^{4-8}$ Even though we consider a time dependent, stochastic effective body force, we still concentrate in this paper on the conduction dominated limit, leaving the study of convection dominated transfer for future work. In this limit, and under constant acceleration and moderate values of the Rayleigh number, the flow inside the cavity is known to reach a steady state. ${ }^{9,10}$ The initial transient regime away from an initial quiescent state has also been studied and is discussed in reference. ${ }^{4}$ It is found there that as the strength of acceleration increases, the transient flow goes from an essentially overdamped decay to a damped oscillatory decay, both leading to the asymptotic steady state.

Concerning time dependent acceleration fields, previous analyses of the effect of $g$-jitter on cavity flow have consid- 
ered effective acceleration fields that are either periodic in time or a sequence of isolated pulses of short duration. ${ }^{11-17}$ Jacqmin ${ }^{16}$ studied the stability of a stratified fluid layer in a periodic acceleration field, either parallel or perpendicular to the density gradient. He addressed the linear stability of a base state in which the velocity field oscillated in time with the same frequency as the imposed acceleration field. More recently, Alexander et al. ${ }^{13}$ studied, by numerical means, natural convection in a directional solidification cell. Although they explicitly considered coupled heat and mass transport, and boundary conditions appropriate for that case (non-zero mass fluxes at two of the boundaries), some of the flows obtained are qualitatively similar to those studied here. The effective acceleration field in their case was modeled as a linear combination of periodic functions of different frequencies and as a sequence of isolated pulses of short duration. Their results imply that substantial convective flow is promoted when the acceleration field is normal to the dominant density gradient, and they analyzed the resulting compositional inhomogeneities in the growing crystal phase.

The approach that we have chosen involves modeling $g$-jitter by a stochastic process, that is, by a sequence of random acceleration field values. The particular stochastic process used is known as narrow band noise and is characterized by three parameters: a mean squared intensity of the fluctuations $G^{2}$, a characteristic frequency $\Omega$, and a correlation time $\tau$. The associated power spectrum is a Lorentzian function of the angular frequency $\omega$, centered at $\omega=\Omega$ and of width $1 / \tau$. This power spectrum provides a reasonable fit to power spectra measured in microgravity environments. To completely define the stochastic process, higher order multitime correlations have to be specified. Given the lack of information on these quantities, the most reasonable a priori choice is to define the higher order multi-time statistics as Gaussian. This choice is based on the assumption that numerous independent sources are simultaneously contributing to the effective acceleration field. In any event, the study that we present in this paper involves only up to second moments and therefore only considers up to two-time correlations of the noise. Up to second moments, each realization of narrow band noise can be viewed as a temporal sequence of periodic functions of angular frequency $\Omega$, with amplitude and phase that remain constant only for a finite amount of time ( $\tau$ on average). At random intervals new values of the amplitude and phase are drawn from prescribed distributions. This interpretation is based on the following mechanism underlying the residual acceleration field: a natural vibration frequency of the spacecraft structure $\Omega$, is excited by a series of mechanical disturbances inside the spacecraft, these disturbances being of random amplitude and occurring at a sequence of unknown (and essentially random) instants of time. ${ }^{1}$

From a theoretical standpoint, narrow band noise provides a convenient way of interpolating between monochromatic noise (akin to the traditional studies involving a deterministic and periodic acceleration field) and white noise (in which no frequency component is preferred). Narrow band noise reduces to (Gaussian) white noise of strength $D$ as $\tau \rightarrow 0$ with $D=G^{2} \tau$ finite; whereas, for $\tau \rightarrow \infty$ with $G^{2}$ finite, monochromatic noise is recovered.

In Sec. II, a simple analytic calculation is presented to study the onset of convective flow under both deterministic and stochastic acceleration fields. In the deterministic case, the vorticity field is oscillatory with an amplitude that remains bounded, even in the absence of viscous dissipation. When a stochastic acceleration field is considered instead, the mean squared value of the vorticity field grows linearly in time, until viscous dissipation causes it to saturate at a stationary value. In Sec. III, we describe a numerical algorithm designed to integrate the coupled equations governing fluid motion and heat diffusion explicitly allowing for a narrow band noise as the body force. In Sec. IV, we present the results obtained by numerical integration of the system of equations, and compare them with the predictions of the analytic calculations for a wide range of both fluid and noise parameters.

\section{CAVITY FLOW}

\section{A. General formulation and scaling}

We consider a Boussinesq fluid enclosed in a laterally heated, two dimensional cell of side $L$. The initial condition at $t=0$ is a quiescent fluid with a uniform temperature gradient along the $x$ direction. The two walls perpendicular to the $x$-direction are heat conducting whereas the other two walls are adiabatic (Fig. 1). We assume that the equation of state of the fluid is given by $\rho=\rho_{0}\left[1+\alpha\left(T-T_{0}\right)\right]$, where $\rho$ is the mass density of the fluid at temperature $T, \rho_{0}$ is a reference density at some temperature $T_{0}$, and $\alpha$ is the thermal expansion coefficient. At $t=0$, a time dependent acceleration field is turned on. The component of the acceleration field parallel to the initial density gradient is too weak in typical microgravity conditions to trigger any fluid motion. The coupling between this component and the fluid variables that describe the displacement away from the quiescent state is nonlinear (i.e., multiplicative in the stochastic sense). With respect to this component, the quiescent state is a stationary solution which may be stable or unstable depending on whether the Rayleigh number exceeds a finite threshold value. The multiplicative coupling implies that a time dependent acceleration field may lead to shifts in the threshold for convective instability, and also new resonance phenomena may occur. However, for typical $g$-jitter intensities and typical temperature gradients across the cavity, ordinary Rayleigh-Bénard or Rayleigh-Taylor instabilities are not likely to occur. We have chosen to neglect this component in our initial study. On the other hand, components of the acceleration field perpendicular to the initial density gradient need not exceed a finite threshold to induce convection. However, the effective acceleration is weak and averages virtually to zero, raising the questions of whether a significant convective flow can be generated, and how it depends on the parameters of $g$-jitter. For the purposes of the analysis presented in this paper, we take the average of the acceleration field to be zero. If $g(t)$ does not average to zero, the flow resulting from its average value would be the same as that of the classical work involving a constant gravity. ${ }^{17}$ 
The starting point of our analysis is the two dimensional stream function-vorticity formulation of the Navier-Stokes equations in the Boussinesq approximation. The incompressibility condition is expressed, $\nabla \cdot \mathbf{v}=0$ where $\mathbf{v}=(u, v)$ is the fluid vclocity. The streamfunction $\psi$, is defined according to

$$
u=\frac{\partial \psi}{\partial y}, \quad v=-\frac{\partial \psi}{\partial x},
$$

while the vorticity is defined by.

$$
\xi=\frac{\partial v}{\partial x}-\frac{\partial u}{\partial y}
$$

Combining the previous two equations yields a Poisson equation for the streamfunction with the vorticity as a source distribution,

$$
\nabla^{2} \psi=-\xi
$$

The equation governing the evolution of the vorticity field is

$$
\frac{\partial \xi}{\partial t}+\nabla \cdot(\mathbf{v} \xi)=\nu \nabla^{2} \xi-\alpha \frac{\partial T}{\partial x} g(t),
$$

where $\nu$ is the kinematic viscosity of the fluid. We model $g(t)$ by a narrow band noise defined by,

$$
g(t)=S_{1}(t) \cos \Omega t+S_{2}(t) \sin \Omega t,
$$

such that $S_{1}(t)$ and $S_{2}(t)$ are two independent, OrnsteinUhlenbeck processes satisfying

$$
\left\langle S_{i}\right\rangle=0, \quad\left\langle S_{i}(t) S_{j}\left(t^{\prime}\right)\right\rangle=G^{2} \delta_{i j} e^{-\left|t-t^{\prime}\right| / \tau}, \quad i=1,2,
$$

where $G^{2}$ is the intensity of the noise, $\tau$ the correlation time and $\delta_{i j}$ is the Kronecker delta. Each Ornstein-Uhlenbeck process can be obtained from

$$
\frac{d S_{i}}{d t}=-\frac{S_{i}}{\tau}+\frac{\eta_{i}(t)}{\tau},
$$

where $\eta_{i}(t)$ are two independent Gaussian, white noise processes defined by

$$
\left\langle\eta_{i}\right\rangle=0, \quad\left\langle\eta_{i}(t) \eta_{j}\left(t^{\prime}\right)\right\rangle=2 D \delta_{i j} \delta\left(t-t^{\prime}\right),
$$

with $D=G^{2} \tau$. Therefore, the usual set of equations governing fluid motion has to be supplemented in this case with Eqs. (5) and (7).

The heat diffusion equation is

$$
\frac{\partial T}{\partial t}+\nabla \cdot(\mathbf{v} T)=\kappa \nabla^{2} T,
$$

where $\kappa$ is the thermal diffusivity. The boundary conditions on the temperature are $T$ constant on the side walls of the cavity where the temperature is fixed, and $\partial T / \partial y=0$ on the two insulating side walls. The streamfunction satisfies $\psi=0$ on the four side walls, and the boundary conditions on the vorticity are derived from the governing equalions subject to the condition that the normal and tangential components of the velocity equal zero, consistent with the assumed no-slip boundary conditions.

Before we proceed with the scaling of the equations, it is useful to explicitly consider two limits of narrow band noise.
The power spectrum $P(\omega)$ is the Fourier transform of the auto-correlation function of $g(t)$. It can be computed from Eqs. (5) and (6). We find,

$$
P(\omega)=\frac{G^{2} \tau}{2 \pi}\left(\frac{1}{1+\tau^{2}(\omega-\Omega)^{2}}+\frac{1}{1+\tau^{2}(\omega+\Omega)^{2}}\right) .
$$

The white noise limit of narrow band noise corresponds to taking $\tau \rightarrow 0$, while keeping $D=G^{2} \tau$ finite. Hence $\lim _{\tau \rightarrow 0} P(\omega)=D / \pi$. In the opposite limit, $\tau \rightarrow \infty$ with $G^{2}$ finite monochromatic noise is recovered with $\lim _{\tau \rightarrow \infty} P(\omega)=\left(G^{2} / 2\right)[\delta(\omega-\Omega)+\delta(\omega+\Omega)]$. Monochromatic noise can be compared to a deterministic function $g(t)=g_{0} \cos \Omega t$. By defining the second moment of the deterministic function $\left\langle g^{2}\right\rangle=(1 / T) \int_{0}^{T} g^{2}(t) d t$, we find $\left\langle g^{2}\right\rangle=g_{0}^{2} / 2$.

To introduce dimensionless variables, we rescale lengths by $L$, times by $L^{2} / \kappa$, temperatures by $\Delta T$ (the imposed temperature difference across the cavity), and acceleration by $\sqrt{2 G^{2} \tau \kappa / L}$. Note that we have introduced the noise correlation time explicitly in the scaling of the acceleration. This is a convenient scaling in the stochastic case, since in the limit of small $\tau$ the intensity of the stochastic forcing is really given by $G^{2} \tau$. From now on, all variables will be dimensionless and all physical constants will be dimensional. When confusion may arise, we superscript dimensional variables with an * (e.g., $\left.g_{0}^{*}, G^{*}, \Omega^{*}, \tau^{*}\right)$.

In dimensionless variables the complete set of equations reads,

$$
\begin{aligned}
& u=\frac{\partial \psi}{\partial y}, \quad v=-\frac{\partial \psi}{\partial x}, \\
& \nabla^{2} \psi=-\xi \\
& \frac{\partial \xi}{\partial t}+\nabla \cdot(\xi \mathbf{v})=\operatorname{Pr} \nabla^{2} \xi-\mathrm{R} \sqrt{\operatorname{Pr}} \frac{\partial T}{\partial x} g(t), \\
& \frac{\partial T}{\partial t}+\nabla \cdot(T \mathbf{v})=\nabla^{2} T,
\end{aligned}
$$

where we have defined the Prandtl number $\operatorname{Pr}=\nu / \kappa$, and a Rayleigh number appropriale to the stochastic case,

$$
\mathrm{R}=\frac{\alpha \Delta T L^{2} \sqrt{2 G^{* 2} \tau^{*}}}{\kappa \sqrt{\nu}} .
$$

We will see that, with the above definition, the stochastic Rayleigh number $\mathbf{R}$ plays the role of the usual Rayleigh number Ra for constant acceleration $g_{0}^{*}$ defined as

$$
\mathrm{Ra}=\frac{\alpha \Delta T L^{3} g_{0}^{*}}{\kappa \nu} .
$$

These equations have to be supplemented by the equations defining the noise. In dimensionless variables, we have

$$
g(t)=S_{1}(t) \cos \Omega t+S_{2}(t) \sin \Omega t,
$$

such that $S_{1}(t)$ and $S_{2}(t)$ are two independent OrnsteinUhlenbeck processes satisfying,

$$
\left\langle S_{i}(t) S_{j}\left(t^{\prime}\right)\right\rangle=\frac{1}{2 \tau} \delta_{i j} e^{-\left|t-t^{\prime}\right| / \tau},
$$




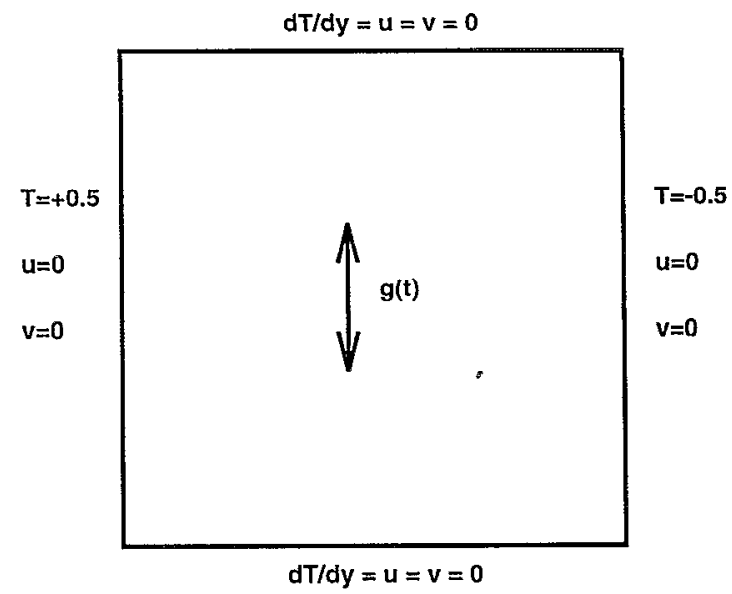

FIG. 1. Configuration of the cavity. Boundary conditions for the dimensionless variables are also shown.

which can be obtained from white noise satisfying $\left\langle\eta(t) \eta\left(t^{\prime}\right)\right\rangle=\delta\left(t-t^{\prime}\right)$. In dimensionless units, the power spectrum of $g(t)$ is,

$$
P(\omega)=\frac{1}{4 \pi}\left(\frac{1}{1+\tau^{2}(\omega-\Omega)^{2}}+\frac{1}{1+\tau^{2}(\omega+\Omega)^{2}}\right),
$$

which reduces to $P=1 / 2 \pi$ in the white noise limit, and to $P(\omega)=(1 / 4 \pi)(\delta(\omega-\Omega)+\delta(\omega+\Omega))$ in the monochromatic limit.

The reduction of Eq. (13) to include a deterministic forcing, $g^{*}(t)=g_{0}^{*} \cos \Omega t$, must be accomplished as follows. Rescaling $g_{0}^{*}$ by the previously defined stochastic acceleration scale, $\sqrt{2 G^{* 2} \tau^{*} \kappa} / L$, we obtain $g(t)=(1 / \sqrt{\tau}) \cos \Omega t$ $=\left(L / \sqrt{\tau^{*} \kappa}\right) \cos \Omega t$. Now the forcing term in Eq. (13) reduces to $\mathrm{R} \sqrt{\operatorname{Pr}} g(t)=\operatorname{Ra} \operatorname{Pr} \cos \Omega t$, with the identification $g_{0}^{* 2} \equiv 2 G^{* 2}$.

\section{B. Early time asymptotic behavior}

We present here a simplified analytic description of the onset of convection in a large cavity highlighting those aspects of the flow peculiar to a stochastic model of the effective acceleration; these effects would be absent were $g(t)$ a deterministic function. We focus first on the transient behavior away from the quiescent state at $t=0$ in a sufficiently large cavity such that the vorticity field is almost uniform. At early times (shortly after the acceleration field is turned on), the convective term in Eq. (4) can be neglected so that,

$$
\frac{\partial \xi}{\partial t}=\operatorname{Pr} \nabla^{2} \xi-\operatorname{R} \sqrt{\operatorname{Pr}} g(t) \frac{\partial T}{\partial x} .
$$

Furthermore, at these early times the body force will balance inertia yielding,

$$
\xi(t) \simeq-\mathrm{R} \sqrt{\operatorname{Pr}} \int_{0}^{t} d s g(s),
$$

where we have also assumed that the variation of the linear temperature profile is negligible ( $\partial T / \partial x \approx 1$ in dimensionless units). The region of validity of this early time approximation can be found in the scaling of the full equation. Were $g(t)$ a constant, $g_{0}$, the vorticity would reach a maximum value of order $\xi_{\text {sat }} \sim \mathrm{Ra}$, with Ra given by Eq. (16), since the viscous term would be of order Pr. Hence, Eq. (21) is expected to be a valid approximation as long as $\xi \ll \xi_{\text {sat }}$. In the stochastic case the same considerations apply, now with the stochastic Rayleigh number R defined by Eq. (15) giving the scale of saturation of vorticity, $\xi_{\text {sat }} \sim \mathrm{R}$, as we will see in Sec. $\mathrm{V}$.

\section{Periodic g-jitter}

For the purpose of comparing the flow resulting from a stochastic acceleration to a standard deterministic one, we first consider the case of periodic acceleration modulation, $g^{*}(t)=g_{0}^{*} \cos (\Omega t+\phi)$. Substitution of $g(t)$ $=\left(L / \sqrt{\tau^{*} \kappa}\right) \cos (\Omega t+\phi)$ into Eq. (21), yields for $\xi(0)=0$,

$$
\xi(t)=\frac{\operatorname{RaPr}}{\Omega}[\sin \phi-\sin (\Omega t+\phi)] .
$$

As discussed above, this solution will be valid when $\xi \ll \xi_{\text {sat }}$, or when $\Omega / \mathrm{Pr}=1 / \operatorname{Re} \ll 1$, where we have defined $\mathrm{Re} \equiv \Omega^{*} / \nu / \mathrm{L}^{2}$. This number plays the role of a Reynolds number and was defined in Ref. 15. One important feature of Eq. (22) is that there is a constant contribution to the vorticity if $\sin \phi \neq 0$. Thus, although $g^{*}(t)$ averages to zero, there will be net flow. Of course, one may argue that such an effect reflects a pathological feature of modeling $g^{*}(t)$ by a periodic function, since there is no particular reason to choose any given value of the phase $\phi$.

Before proceeding with the stochastic calculation which is itself based on Eq. (22), we note that even the simple approximation given in Eq. (22) captures some of the features of cavity flow under a periodically modulated acceleration field. Equation (22) can be compared with numerical calculations by Alexander et al. ${ }^{13}$ who studied the onset of natural convection in cells appropriate for crystal growth by the Bridgman-Stockbarger method. Their container had $L=1$ $\mathrm{cm}$ and a temperature difference across the lateral walls of $115 \mathrm{~K}$. The fluid was assumed to have the following physical properties: $\nu=1.3 \times 10^{-3} \mathrm{~cm}^{2} / \mathrm{s}, \alpha=2.5 \times 10^{-4} \mathrm{~K}^{-1}$ and the thermal diffusivity $\kappa=0.13 \mathrm{~cm}^{2} / \mathrm{s}$. In one case $g_{0}^{*}=10$ $\mathrm{cm} / \mathrm{s}^{2}$ with $\Omega^{*}=2 \pi \mathrm{s}^{-1}$, or $\operatorname{Re} \approx 5000$. From Eq. (22) and the given parameter values, we obtain $\xi_{\max }^{*}=\max \left\{\xi^{*}(t)\right\}$ $=0.05 \mathrm{~s}^{-1}$. The maximum velocity of the fluid in our approximation is found near the walls and equals $u_{\max }^{*} \approx \xi_{\max }^{*} L / 2=0.025 \mathrm{~cm} / \mathrm{s}$. From Figs. 10 and 11 of their work, the maximum velocity obtained numerically is $u_{\text {max }}^{*} \approx 0.02 \mathrm{~cm} / \mathrm{s}$, in good agreement with our estimate. We further note that there is a phase difference of $\pi / 2$ between $g^{*}(t)$ and $\xi^{*}(t)$ [Eq. (22)] in the limit considered. This also agrees with the numerical calculations shown in Figs. 10 and 11 of Alexander et al. As expected, our estimate becomes worse at smaller values of Re. In another calculation in the same work, the values $g_{0}^{*}=0.01 \mathrm{~cm} / \mathrm{s}^{2}$ and $\Omega^{*}=2 \pi \times 10^{-3} \mathrm{~s}^{-1}$ were used. Our estimate for this case is $u_{\text {max }}^{*}=0.025 \mathrm{~cm} / \mathrm{s}$, whereas the value obtained numerically is $u_{\max }^{*} \approx 0.001 \mathrm{~cm} / \mathrm{s}$. Here, however, $\operatorname{Re} \approx 5$. For this smaller valuc of $\mathrm{Re}$, we expect our simple estimate to break down, as well as $g^{*}(t)$ and $\xi^{*}(t)$ to be closer to being in phase. From 
Fig. 2 in Alexander et al., we note that both quantities indeed change in phase, implying that viscous effects cannot be neglected.

Unless $\phi=0$, the oscillatory motion of the fluid is superimposed to a systematic drift proportional to $\sin \phi$. Even though $g^{*}(t)$ averages to zero, the symmetry between clockwise and counterclockwise rotation is broken by the initial phase, and a mean rotation of the fluid follows. Such a dependence on the initial phase is clearly an artifact of having considered a strictly deterministic and periodic $g$-jitter. Even within the framework of periodic $g$-jitter, a sensible first approximation accounting for the stochastic nature of the microgravity environment is to assume that the phase $\phi$ is a random variable, perhaps uniformly distributed in $[0,2 \pi)$. Thus, all quantities can be averaged over the probability distribution of the variable $\phi$. Averaging Eq. (22) over uniform distribution of $\phi$ restores the symmetry $\xi \rightarrow-\xi$ and gives

$$
\langle\xi\rangle=0, \quad\left\langle\xi^{2}(t)\right\rangle \simeq\left(\frac{\operatorname{RaPr}}{\Omega}\right)^{2} \sin ^{2}\left(\frac{\Omega t}{2}\right) .
$$

In this approximation, the root mean squared vorticity remains bounded and equals the amplitude of the oscillation in Eq. (22). Considering such an average over initial phases is similar, though not identical to, the results which would be obtained for a process known as "monochromatic noise." The fact that, even in the absence of viscous dissipation, the vorticity saturates at a finite value must be contrasted with the unbounded growth obtained in the following section.

\section{Narrow band noise}

Combining the narrow band noise model of $g$-jitter with Eq. (21), yields an expression for the second moment of the vorticity as a function of time. Formally, we can obtain this second moment by squaring Eq. (21) and taking the ensemble average,

$$
\left\langle\xi^{2}(t)\right\rangle=\mathbf{R}^{2} \operatorname{Pr} \int_{0}^{t} d s \int_{0}^{t} d s^{\prime}\left\langle g(s) g\left(s^{\prime}\right)\right\rangle .
$$

The correlation function for narrow band noise, $\left\langle g(t) g\left(t^{\prime}\right)\right\rangle$, is easily obtained by combining Eqs. (5) and (18) to yield

$$
\left\langle g(t) g\left(t^{\prime}\right)\right\rangle=\frac{1}{2 \tau} e^{-\left|t-t^{\prime}\right| / \tau} \cos \Omega\left(t-t^{\prime}\right) .
$$

Combining the two preceding equations results in the following form for the second moment of $\xi(t)$ :

$$
\left\langle\xi^{2}(t)\right\rangle=\mathrm{R}^{2} \operatorname{Pr} F(t),
$$

with

$$
\begin{aligned}
F(t)= & \frac{t}{1+\tau^{2} \Omega^{2}}+\frac{1}{\tau\left(\tau^{-2}+\Omega^{2}\right)^{2}} \\
& \times\left(\left(\Omega^{2}-\tau^{-2}\right)\left(1-e^{-t / \tau} \cos \Omega t\right)\right. \\
& \left.-\frac{2 \Omega}{\tau} e^{-t / \tau} \sin \Omega t\right) .
\end{aligned}
$$

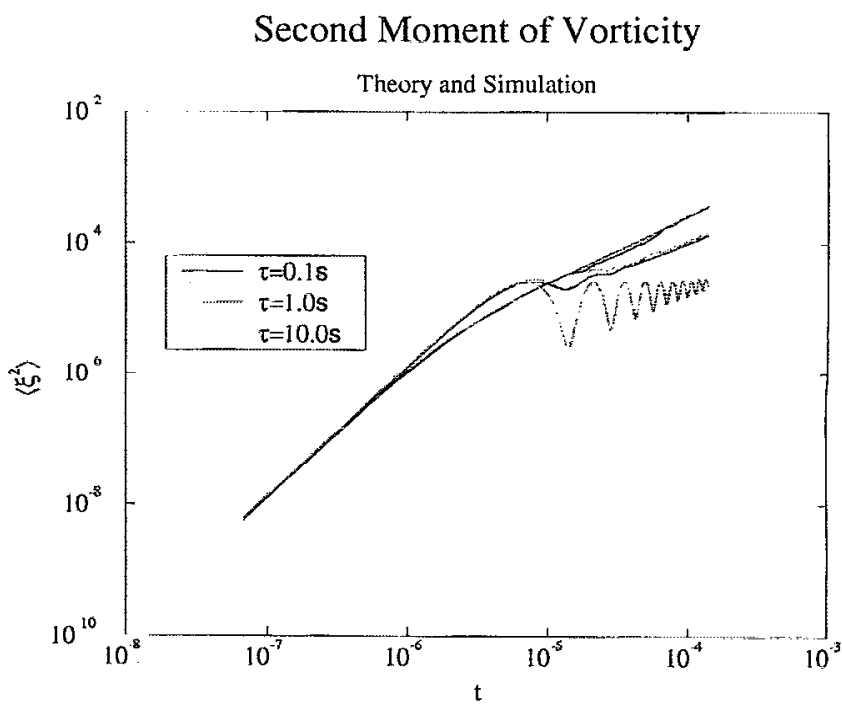

FIG. 2. The second moment of the vorticity as a function of time for a fixed frequency of $f^{*}=1 \mathrm{~Hz}$, which corresponds to $f=7.1 \times 10^{4}$, in dimensionless units. The corresponding dimensionless values for $\tau$ are $1.4 \times 10^{-6}(R$ $=0.72), 1.4 \times 10^{-5}(R=2.2)$, and $1.4 \times 10^{-4}(R=7.2)$. The analytical prediction, Eq. (26), is plotted over the results of the numerical solutions.

This equation is the central result of our analysis: $F(t)$ is a linearly increasing function of time with an oscillatory component. The amplitude of the oscillatory component decays exponentially with a decay rate of $1 / \tau$. This function is shown in Figs. 2 and 3 for several values of the noise parameters. Because the average vorticity vanishes and the mean squared vorticity increases linearly in time, the early time behavior of the vorticity in the cavity is analogous to a random walk. We must remark, however, that the unbounded linear growth of $\left\langle\xi^{2}\right\rangle$ in Eq. (26) results from neglecting vis-

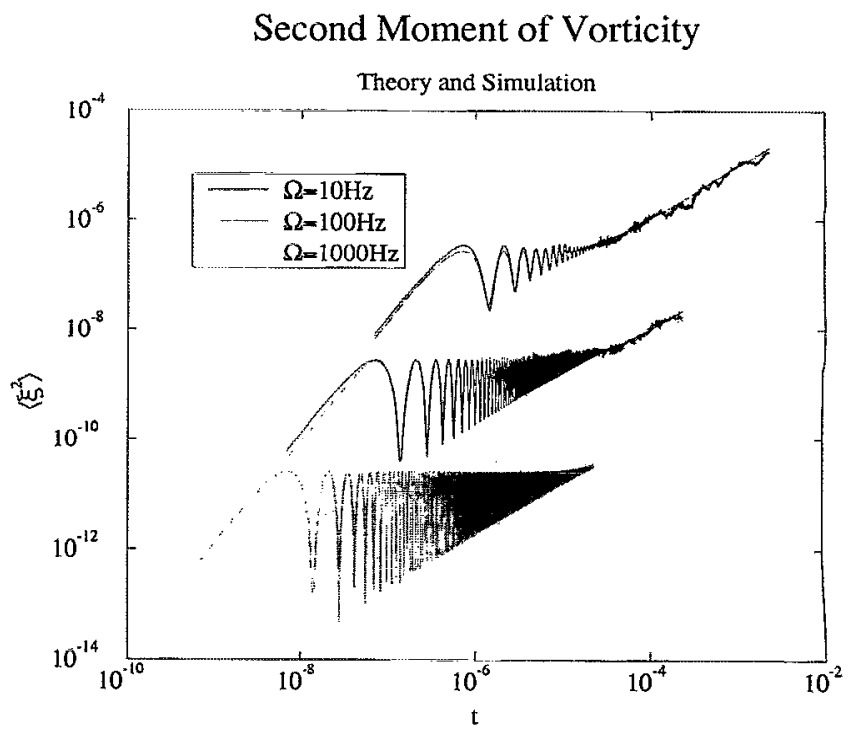

FIG. 3. The second moment of the vorticity as a function of time for a fixed correlation time $\tau^{*}=1 \mathrm{~s}$, which corresponds to $\tau=1.4 \times 10^{-5}(R=2.2)$, in dimensionless units. The corresponding dimensionless values for $f$ are $7.1 \times 10^{5}, 7.1 \times 10^{6}$, and $7.1 \times 10^{7}$. The analytical prediction, Eq. (26), is plotted over the results of the numerical solutions. 
cous dissipation in our calculation. As stated above, this approximation is expected to be valid only when $\xi \ll \xi_{\text {sat }} \sim \mathrm{R}$. In Sec. IV, the results from a numerical solution to the full system of equations will be compared to our analytical results. It turns out that our approximation agrees with the numerical calculations over a wide range of parameters.

\section{NUMERICAL INTEGRATION}

In order to ascertain the validity of the simple analytical arguments given above and to verify the predictions of Eq. (26), we have undertaken a numerical solution of the NavierStokes equations in the Boussinesq approximation. Given that there exists limited experience in solving the NavierStokes equations under the effects of a stochastic body force, in this study we chose to use a simple finite difference technique. The method used is the Forward Time-Centered Space (FTCS) method, which is first order accurate in time and second order accurate in space. Although this method is known to have a variety of undesirable stability problems, our calculations have been performed wcll within acccptable stability parameters. ${ }^{18}$ Without a diffusion term in the momentum equation, Eq. (4), the FTCS method is unconditionally unstable, but with a diffusion term the method becomes conditionally stable. The small Rayleigh numbers and relatively large Prandtl numbers discussed in this paper are well within the limits of stability of the method.

The spatial discretization of Eq. (13) is straightforward, but the temporal discretization requires more care, as discussed in Ref. 19 for the simpler case of a parametrically driven harmonic oscillator. We begin with Eq. (13) at time, $t$, where the state of the fluid is completely known and integrate the equation from $t$ to $t+\Delta t$,

$$
\begin{aligned}
& \int_{t}^{t+\Delta t} d t^{\prime} \frac{\partial \xi\left(t^{\prime}\right)}{\partial t^{\prime}}+\int_{t}^{t+\Delta t} d t^{\prime} \nabla \cdot\left[\mathbf{v}\left(t^{\prime}\right) \xi\left(t^{\prime}\right)\right] \\
& =\operatorname{Pr} \int_{t}^{t+\Delta t} d t^{\prime} \nabla^{2} \xi\left(t^{\prime}\right)-\mathrm{R} \sqrt{\operatorname{Pr}} \int_{t}^{t+\Delta t} d t^{\prime} g\left(t^{\prime}\right) \frac{\partial T\left(t^{\prime}\right)}{\partial x}
\end{aligned}
$$

The first term is integrated exactly, while the remaining terms not involving $g(t)$ are expanded in a Taylor series around $t$. Hence, we obtain to first order,

$$
\begin{aligned}
\xi(t+\Delta t)-\xi(t)= & -\nabla \cdot[\mathbf{v}(t) \xi(t)] \Delta t+\operatorname{Pr} \nabla^{2} \xi(t) \Delta t \\
& -\mathrm{R} \sqrt{\operatorname{Pr}} \frac{\partial T(t)}{\partial x} \int_{t}^{t+\Delta t} d t^{\prime} g\left(t^{\prime}\right) .
\end{aligned}
$$

The remaining integral of $g(t)$, from $t$ to $t+\Delta t$ is itself a random variable, which we denote by $\Gamma(t)$. This term must be handled separately. The relevant details on the evaluation of this stochastic integral are given in the Appendix.

The spatial discretization follows standard procedure. ${ }^{18}$ The functions $(\xi, \psi, T, u, v)$ are evaluated at the $n_{x} \times n_{y}$ nodes of the evenly spaced grid. The difference equation for the momentum equation at node $i, j$ is written:

$$
\begin{aligned}
\frac{\xi_{i, j}^{t+\Delta t}-\xi_{i, j}^{t}}{\Delta t} & -\frac{u_{i+1, j}^{t} \xi_{i+1, j}^{t}-u_{i-1, j}^{t} \xi_{i-1, j}^{t}}{2 \Delta x} \\
& -\frac{v_{i, j+1}^{t} \xi_{i, j+1}^{t}-v_{i, j-1}^{t} \xi_{i, j-1}^{t}}{2 \Delta y} \\
& +\operatorname{Pr}\left(\frac{\xi_{i+1, j}^{t}+\xi_{i-1, j}^{t}-2 \xi_{i, j}^{t}}{\Delta x^{2}}+\frac{\xi_{i, j+1}^{t}+\xi_{i, j-1}^{t}-2 \xi_{i, j}^{t}}{\Delta y^{2}}\right) \\
& \quad-\frac{\mathrm{R} \sqrt{\operatorname{Pr}}}{\Delta t} \Gamma(t) \frac{T_{i+1, j}^{t}-T_{i-1, j}^{t}}{2 \Delta x}
\end{aligned}
$$

and the heat diffusion equation is written:

$$
\begin{aligned}
& \frac{T_{i, j}^{t+\Delta t}-T_{i, j}^{t}}{\Delta t} \\
& =-\frac{u_{i+1, j}^{t} T_{i+1, j}^{t}-u_{i-1, j}^{t} T_{i-1, j}^{t}}{2 \Delta x} \\
& \quad-\frac{v_{i, j+1}^{t} T_{i, j+1}^{t}-v_{i, j-1}^{t} T_{i, j-1}^{t}}{2 \Delta y} \\
& \quad+\frac{T_{i+1, j}^{t}+T_{i-1, j}^{t}-2 T_{i, j}^{t}}{\Delta x^{2}}+\frac{T_{i, j+1}^{t}+T_{i, j-1}^{t}-2 T_{i, j}^{t}}{\Delta y^{2}}
\end{aligned}
$$

Note that the advection terms have been kept in conservative form. The Poisson equation for the streamfunction, Eq. (3), is solved using the Successive Over Relaxation technique (SOR) ${ }^{20}$ which solves the associated discrete version of a diffusion equation, $\partial \psi / \partial t+\nabla^{2} \psi=-\xi$. If the equation reaches a steady state, $\partial \psi / \partial t=0$, the Poisson equation is satisfied. The no-slip boundary conditions are implemented as follows: for the vorticity, the derived first-order form, $\xi_{\text {wall }}=-2 \psi_{\text {wall }+1} / \Delta x^{2}$, is used, where the " +1 " indicates "toward the interior"; 18 for the streamfunction we have simply $\psi_{\text {wall }}=0$. For the fixed temperature boundary conditions we have $T=-1 / 2$ at $x=0$ and $T=1 / 2$ at $x=1$, and for the insulating boundary condition we use $T_{\text {wall }}=T_{\text {wall }+1}$.

\section{NUMERICAL RESULTS}

All the calculations presented in this paper were performed on a $32 \times 32$ grid, with the following parameters: $\nu=1.0 \times 10^{-2} \quad \mathrm{~cm}^{2} / \mathrm{s} ; \quad \kappa=1.4 \times 10^{-3} \mathrm{~cm}^{2} / \mathrm{s} ; \quad \Delta T=15 \quad \mathrm{~K} ;$ $\alpha=2.6 \times 10^{-4} \mathrm{~K}^{-1}$; and, $L=10 \mathrm{~cm}$. The stochastic Rayleigh number, $\mathrm{R}$, is given by $\mathrm{R}=\mathrm{Ra} \sqrt{2 \tau \operatorname{Pr}}$. The conditions at $t=0$, when the fluctuating acceleration is turned on, are $\psi=\xi=0$ and the temperature distribution was a constant gradient in the $x$-direction. Usually, $\Delta t^{*}$ was taken at $5 \times 10^{-3} \mathrm{~s}$, though smaller values (such that $1 / f^{*}=2 \pi / \Omega^{*} \approx 20 \Delta t^{*}$ ) were used for higher frequencies. In the results presented here, $G^{* 2}=6.0 \times 10^{-4} \mathrm{~cm}^{2} \mathrm{~s}^{-4}$, though a broad range of values have been tested. This value is an estimate obtained from shuttle data. The values of the physical parameters given above correspond to $\mathrm{Ra}=163$ and $\operatorname{Pr}=7.1$. Finally, an important distinction with the case of 
periodic acceleration field is that the stochastic nature of the acceleration field requires that many distinct runs involving independent realizations of the random function be considered, and the appropriate averages calculated over this ensemble. The results presented in this paper are based on averages over an ensemble of 300 independent realizations.

For the values of the parameters given above, the temperature distribution in the cavity is not appreciably modified by the flow. Also, since viscous dissipation is mainly confined to boundary layers adjacent to the walls, the vorticity field remains nearly uniform throughout the cell. With these considerations in mind, the flow can be conveniently characterized by the amplitude of a mode of uniform vorticity spanning almost the entire cavity. Hence we present our results only for the value of the vorticity field at the center of the cavity.

Figure 2 shows the second moment of the vorticity at the center of the cavity, $\left\langle\xi^{2}\right\rangle$, as a function of time, for a fixed frequency $\Omega$ and three different values of the correlation time. For times $t<\tau$, an oscillatory behavior of angular frequency $\Omega$ is observed, most obviously in the lowest curve, which corresponds to the largest correlation time. The amplitude of the oscillation decays approximately exponentially over a period of $\tau$. The uppermost curve shows no evidence of oscillation, since the correlation time of the noise is ten times smaller than the period of oscillation. This approaches the limit of white noise. For times $t>\tau,\left\langle\xi^{2}\right\rangle$ is linear in time. The expressions given in Eq. (26) are superimposed on the numerical result, for the appropriate values of the parameters.

These general features can also be observed as we vary the frequency of the acceleration forcing and keep the correlation time constant. Figure 3 shows the results for three different frequencies. The amplitude of the oscillation decays exponentially, the decay rate being the same for the three curves. The period of oscillation however is different as we have varied the value of $\Omega$. Also, the average slope of each curve is proportional to $1 / \Omega^{2}$.

\section{ESTIMATE OF VISCOUS EFFECTS}

As was discussed above, the average squared vorticity is not expected to increase indefinitely in the stochastic case but will saturate due to viscous dissipation [which was neglected in Eq. (21)]. A quantitative analytic study of the saturation is quite involved. However, a qualitative study possible as long as the motion of the fluid is such that the Laplacian of the vorticity can be considered to remain uniform, permitting us to assume that the effect of the viscous diffusion term in Eq. (21) is simply that of a damping term. Furthermore, we neglect any nonlinear terms in the equation of motion of the vorticity. We obtain

$$
\frac{\partial \xi}{\partial t}=-\operatorname{Pr} \xi+\mathrm{R} \sqrt{\operatorname{Pr}} g(t) .
$$

Equation (31) has the form of a Langevin equation, which is solved to yield

$$
\xi(t)=\xi(0) e^{-\operatorname{Prt}}+\mathrm{R} \sqrt{\operatorname{Pr}} \int_{0}^{t} d s g(s) e^{-\operatorname{Pr}(t-s)} .
$$

We assume that $\xi=0$ at $t=0$ so that $\langle\xi(t)\rangle=0$. In order to obtain $\left\langle\xi^{2}(t)\right\rangle$, we square Eq. (32) and take the ensemble average,

$$
\left\langle\xi^{2}(t)\right\rangle=\mathrm{R}^{2} \operatorname{Pr} \int_{0}^{t} d s \int_{0}^{t} d s^{\prime} e^{-\operatorname{Pr}\left(2 t-s-s^{\prime}\right)}\left\langle g(s) g\left(s^{\prime}\right)\right\rangle,
$$

where the correlation function $\left\langle g(s) g\left(s^{\prime}\right)\right\rangle$ is given by Eq. (25). If we take $g(t)$ to be white noise, such that $\langle g(t)\rangle=0$ and $\left\langle g(t) g\left(t^{\prime}\right)\right\rangle=2 \delta\left(t-t^{\prime}\right)$, we finally obtain,

$$
\left\langle\xi^{2}(t)\right\rangle=\mathrm{R}^{2}\left(1-e^{-2 \operatorname{Pr} t}\right) .
$$

Thus the saturation value at $t \rightarrow \infty$ for white noise is given by $\left\langle\xi^{2}\right\rangle_{\text {sat }}=\mathrm{R}^{2}$. Note that $\left\langle\xi^{2}\right\rangle_{\text {sat }}$ can be written in the form $(\mathrm{R} \sqrt{\mathrm{Pr}})^{2} / \mathrm{Pr}$, which is the ratio of mean squared forcing to dissipation, essentially a fluctuation-dissipation relation for this simplified system.

This result should be contrasted with the corresponding result for a deterministic system. In the latter case, the average value of $\xi$ does not vanish. When saturation is achieved $(\partial \xi / \partial t \rightarrow 0)$ the viscous term is balanced by the forcing term. This implies that the vorticity is given by $\xi_{\text {sat }}^{2} \sim(\mathrm{RaPr})^{2} /(\mathrm{Pr})^{2}$, instead. Thus, the saturation value is given by the ratio of the square of the forcing term and the square of dissipative term.

For $g(t)$ given by narrow band noise, the mean squared velocity also saturates to a finite value. We use Eq. (33) with $\left\langle g(t) g\left(t^{\prime}\right)\right\rangle$ given by Eq. (25), and obtain after some algebra,

$$
\left\langle\xi^{2}(t)\right\rangle=\frac{(\operatorname{Pr} \tau+1) \mathrm{R}^{2}}{(\operatorname{Pr} \tau+1)^{2}+\Omega^{2} \tau^{2}}+\text { decaying terms. }
$$

The decaying terms vanish exponentially at a rate depending both on $\tau$ and the Prandtl number. The saturation value, however, depends not only on $\mathbf{R}$ and the Prandtl number, but on the parameters of the noise as well.

\section{DISCUSSION AND CONCLUSIONS}

A goal of the present work was to determine whether a fluctuating acceleration field of zero mean can induce macroscopic fluid flow on an otherwise quiescent state, in the cavity configuration described above. We have focused on the particular case in which the fluctuating acceleration field is normal to the imposed temperature gradient. Furthermore, the ranges of the parameters of the fluid and cavity chosen are such that the temperature field is not appreciably distorted by the flow. Hence, our study concentrates on the mechanical response of the fluid to the acceleration field in the limit in which convective heat transport is negligible. Of course, both limitations can be easily relaxed in the numerical scheme, and more complex flows will be addressed in the future.

In our analytical work, we have found that a periodic acceleration field induces a periodic flow of amplitude proportional to the amplitude of the acceleration field. The deterministic response depends on the initial phase of the acceleration field, especially in an inviscid fluid, indicating that numerical studies involving a deterministic and periodic $g$-jitter should explore the dependence of the flow on a distribution of initial phases. We have also studied the case of a 
uniform distribution of initial phases and obtained that the mean squared velocity of the fluid is bounded.

We have also been able to derive important characteristics of cavity flow that are peculiar to the stochastic nature of the acceleration field, and would not follow from a deterministic formulation. Although the imposed acceleration field, and hence the vorticity, averages to zero, the vorticity field itself can be described by a random walk at long times. Its average is zero for all times. For short times $t<\tau,\left\langle\xi^{2}\right\rangle$ oscillates with angular frequency $\Omega$, the characteristic frequency of the noise. The amplitude of oscillation, however, decays approximately exponentially over a time period of order $\tau$. For $t>\tau$, the oscillations in $\left\langle\xi^{2}\right\rangle$ are negligible, and $\left\langle\xi^{2}\right\rangle$ increases linearly in time according to

$$
\left\langle\xi^{2}(t)\right\rangle \propto \frac{\mathrm{R}^{2} \operatorname{Pr}}{1+(\Omega \tau)^{2}} t .
$$

At even longer times, the value of $\left\langle\xi^{2}\right\rangle$ is expected to saturate at a constant value because of viscous dissipation in the fluid. In the stochastic case, the saturation vorticity is proportional to $\mathrm{R}$, which must be contrasted with the deterministic case where the saturation is proportional to Ra. This indicates that the saturation value is partly determined by the characteristics of the noise. A numerical study of the long time saturation as a function of the parameters of the fluid and of the noise is currently under way.

A numerical algorithm to solve the equations governing fluid motion has been developed that explicitly allows for a stochastic body force. We have numerically obtained the flow field in a laterally heated, two dimensional cavity under a fluctuating acceleration field normal to the imposed temperature gradient, modeled by narrow band noise. The numerical solution is in excellent agreement with our approximate analytical calculations, and confirms the diffusive (i.e., sublinear) growth of the velocity field in the cavity.

\section{ACKNOWLEDGMENTS}

We are indebted to Wenbin Zhang for the original algorithm to calculate narrow band noise and for many useful discussions. This work is supported by the Microgravity Science and Applications Division of the NASA under Contract No. NAG3-1284. This work is also supported in part by the Supercomputer Computations Research Institute, which is partially funded by U.S. Department of Energy Contract No. DE-FC05-85ER25000.

\section{APPENDIX: INTEGRATION OF NOISE TERM}

We sketch in this appendix the determination of the stochastic integral $\Gamma(t)$ in Eq. (28). We start with the solution of the Ornstein-Uhlenbeck differential equation [Eq. (7)],

$$
S_{i}(s)=S_{i}(t) e^{-(s-t) / \tau}+\frac{1}{\tau} \int_{t}^{s} d s^{\prime} e^{-\left(s-s^{\prime}\right) / \tau} \eta\left(s^{\prime}\right) .
$$

If we substitute Eq. (17) into the expression for $\Gamma(t)$ [Eq. (28)], we have

$$
\Gamma(t)=\int_{t}^{t+\Delta t} d s\left[S_{1}(s) \cos \Omega s+S_{2}(s) \sin \Omega s\right] .
$$

Let us examine the first term in the integral. Substitution of Eq. (A1) for $S_{1}(s)$ yields for the first term in Eq. (A2),

$$
\begin{array}{rl}
\int_{t}^{t+\Delta t} & d s S_{1}(s) \cos \Omega s \\
= & S_{1}(t) \int_{t}^{t+\Delta t} d s \cos \Omega s e^{-(s-t) / \tau} \\
& +\frac{1}{\tau} \int_{t}^{t+\Delta t} d s \cos \Omega s \int_{t}^{s} d s^{\prime} e^{-\left(s-s^{\prime}\right) / \tau} \eta\left(s^{\prime}\right) .
\end{array}
$$

The second term in Eq. (A3) must be simplified using integration by parts to remove the double integral. Noting that

$$
\begin{aligned}
\frac{d}{d s}\left(\frac{1}{\Omega} \cos \Omega s \int_{t}^{s} d s^{\prime} e^{-\left(s-s^{\prime}\right) / \tau} \eta\left(s^{\prime}\right)\right) \\
=\frac{1}{\Omega} \cos \Omega s \eta(s)-\sin \Omega s \int_{t}^{s} d s^{\prime} e^{\left(s-s^{\prime}\right) / \tau} \eta\left(s^{\prime}\right) \\
\quad-\frac{\cos \Omega s}{\Omega \tau} \int_{t}^{s} d s^{\prime} e^{\left(s-s^{\prime}\right) / \tau} \eta\left(s^{\prime}\right),
\end{aligned}
$$

and that

$$
\begin{aligned}
\frac{d}{d s}\left(\frac{1}{\Omega} \sin \Omega s \int_{t}^{s} d s^{\prime} e^{-\left(s-s^{\prime}\right) / \tau} \eta\left(s^{\prime}\right)\right) \\
=\frac{1}{\Omega} \sin \Omega s \eta(s)+\cos \Omega s \int_{t}^{s} d s^{\prime} e^{\left(s-s^{\prime}\right) / \tau} \eta\left(s^{\prime}\right) \\
\quad-\frac{\sin \Omega s}{\Omega \tau} \int_{t}^{s} d s^{\prime} e^{\left(s-s^{\prime}\right) / \tau} \eta\left(s^{\prime}\right),
\end{aligned}
$$

upon substitution of Eq. (A5) into Eq. (A4), and then Eq. (A4) into Eq. (A3), one obtains a form involving single integrals. The final result is

$$
\begin{aligned}
\int_{t}^{t+\Delta t} d s S_{1}(s) \cos \Omega s= & \left.\frac{S_{1}(t) \tau}{1+\tau^{2} \Omega^{2}}\left\{e^{\Delta t / \tau} \Omega \tau \sin \Omega(t+\Delta t)-\cos \Omega(t+\Delta t)\right]-(\Omega \tau \sin \Omega t-\cos \Omega t)\right\} \\
& +\frac{1}{1+\tau^{2} \Omega^{2}} \int_{t}^{t+\Delta t} d s\left\{(\cos \Omega s-\Omega \tau \sin \Omega s)-e^{(t+\Delta t-s) / \tau}[\cos \Omega(t+\Delta t)\right. \\
& -\Omega \tau \sin \Omega(t+\Delta t)]\} \eta(s) .
\end{aligned}
$$


Therefore, after repeating the analogous integration for the second term in Eq. (A2), we finally obtain an equation which has the form,

$$
\Gamma(t)=A_{1}(t) S_{1}(t)+A_{2}(t) S_{2}(t)+F_{1}(t)+F_{2}(t),
$$

$A_{1}$ and $A_{2}$ are deterministic functions with the form,

$$
\begin{aligned}
A_{1}(t)= & \frac{\tau}{1+\tau^{2} \Omega^{2}}\{\cos \Omega t-\Omega \tau \sin \Omega t \\
& \left.-e^{-\Delta t / \tau}[\cos \Omega(t+\Delta t)-\Omega \tau \sin \Omega(t+\Delta t)]\right\},
\end{aligned}
$$

$$
\begin{aligned}
A_{2}(t)= & \frac{\tau}{1+\tau^{2} \Omega^{2}}\{\sin \Omega t+\Omega \tau \cos \Omega t \\
& \left.-e^{-\Delta t / \tau}[\sin \Omega(t+\Delta t)+\Omega \tau \cos \Omega(t+\Delta t)]\right\}
\end{aligned}
$$

The stochastic terms, $S_{1}$ and $S_{2}$, are simply solutions of the Ornstein-Uhlenbeck differential equation, given by Eq. (A1),

$$
S_{i}(t+\Delta t)=e^{-\Delta t / \tau} S_{i}(t)+G_{i}(t),
$$

where

$$
G_{i}(t)=\frac{1}{\tau} \int_{t}^{t+\Delta t} d t^{\prime} e^{-\left(t+\Delta t-t^{\prime}\right) / \tau} \eta_{i}\left(t^{\prime}\right) .
$$

Note that this equation is also exact, but that $G_{i}(t)$ is a stochastic function; $F_{1}$ and $F_{2}$ are also stochastic functions. Explicitly,

$$
\begin{aligned}
F_{1}(t)= & -\frac{\tau}{1+\tau^{2} \Omega^{2}} \int_{t}^{t+\Delta t} d t^{\prime}\{[\cos \Omega(t+\Delta t) \\
& -\Omega \tau \sin \Omega(t+\Delta t)] e^{-\left(t+\Delta t-t^{\prime}\right) / \tau}-\cos \Omega t^{\prime} \\
& \left.-\Omega \tau \sin \Omega t^{\prime}\right\} \eta_{1}\left(t^{\prime}\right), \\
F_{2}(t)= & \frac{\tau}{1+\tau^{2} \Omega^{2}} \int_{t}^{t+\Delta t} d t^{\prime}\{[\sin \Omega(t+\Delta t) \\
& +\Omega \tau \cos \Omega(t+\Delta t)] e^{-\left(t+\Delta t-t^{\prime}\right) / \tau}-\sin \Omega t^{\prime} \\
& \left.+\Omega \tau \cos \Omega t^{\prime}\right\} \eta_{2}\left(t^{\prime}\right) .
\end{aligned}
$$

Because $G_{i}(t)$ and $F_{i}(t)$ are functionals of the same $\eta_{i}(t)$, they are not statistically independent; $G_{i}(t)$ and $F_{i}(t)$ are obtained from the same random sequence of $\eta_{i}(t)$ between $t$ and $t+\Delta t$. However, $G_{i}(t)$ and $F_{i}(t)$ can be calculated because $\eta_{i}(t)$ is a Gaussian process, and therefore the distribution of its integral should also be Gaussian. That is, if we generate $G_{i}(t)$ using $G_{i}(t)=\sqrt{\left\langle G_{i}^{2}\right\rangle} \zeta(t)$, where $\zeta(t)$ is a Gaussianly distributed random number with $\langle\zeta\rangle=0$ and $\left\langle\zeta^{2}\right\rangle=1$, we must find $F_{i}(t)$ as follows. Assume, $F_{i}(t)=b \sqrt{\left\langle F_{i}^{2}\right\rangle} \zeta(t)$ where $\zeta(t)$ is as above. Then, we can determine $b$ from the cross-correlations of $G_{i}$ and $F_{i}$. This gives $b=\left\langle G_{i} F_{i}\right\rangle / \sqrt{\left\langle F_{i}^{2}\right\rangle\left\langle G_{i}^{2}\right\rangle}$. Therefore, the functions are generated as

$$
G_{i}(t)=\sqrt{\left\langle G_{i}^{2}\right\rangle} \zeta(t),
$$

$$
F_{i}(t)=\frac{\left\langle G_{i} F_{i}\right\rangle}{\sqrt{\left\langle G_{i}^{2}\right\rangle}} \zeta(t)
$$

It is easy to calculate these auto-correlations and crosscorrelations from the forms given above. Here we list the variance of $G_{1}(t)$ and the cross-correlations $\left\langle G_{1}(t) F_{1}(t)\right\rangle$. Similar quantities for $G_{2}(t)$ and $F_{2}(t)$ are obtained from the corresponding terms for $G_{1}(t)$ and $F_{1}(t)$ by replacing $\cos \Omega t \rightarrow \sin \Omega t$ and $\sin \Omega t \rightarrow-\cos \Omega t ;$

$$
\left\langle G_{1}^{2}(t)\right\rangle=\frac{1}{2 \tau}\left\{1-e^{-2 \Delta t / \tau}\right\},
$$

$$
\begin{aligned}
\left\langle G_{1}(t) F_{1}(t)\right\rangle= & \frac{1}{1+\Omega^{2} \tau^{2}}\left\{\left[\cos \Omega(t+\Delta t)-e^{-\Delta t / \tau} \cos \Omega t\right]\right. \\
& -\frac{1}{2}\left(1-e^{-2 \Delta t / \tau}\right)[\cos \Omega(t+\Delta t) \\
& -\Omega \tau \sin \Omega(t+\Delta t)]\} .
\end{aligned}
$$

This completes our calculation. The algorithm can be summarized as follows:

begin:

loop:

$$
\text { calculate } S_{i}(t)
$$

calculate $A_{i}(t)$ from Eq. (A8) calculate $\left\langle G_{i}^{2}(t)\right\rangle$ from Eq. (A13) calculate $\left\langle G_{i}(t) F_{i}(t)\right\rangle$ from Eq. (A14) let $G_{i}(t)=\sqrt{\left\langle G_{i}^{2}(t)\right\rangle} \zeta(t)$ let $F_{i}(t)=\frac{\left\langle G_{i}(t) F_{i}(t)\right\rangle}{\sqrt{\left\langle G_{i}^{2}(t)\right\rangle}} \zeta(t)$ calculate $\Gamma(t)$ from Eq. (A7) calculate $S_{i}(t+\Delta t)$ from Eq. (A9) let $t=t+\Delta t$

\footnotetext{
endloop:

end:
}

\footnotetext{
${ }^{1}$ W. Knabe and D. Eilers, "Low-gravity environment in spacelab," Acta Astronaut. 9, 187 (1992).

${ }^{2}$ G. K. Batchelor, "Heat transfer by free convection across a closed cavity between vertical boundaries at different temperatures," Q. Appl. Math. 12, 209 (1954)

${ }^{3}$ A. E. Gill, "The boundary-layer regime for convection in a rectangular cavity," J. Fluid Mech. 26, 515 (1966).

${ }^{4} \mathrm{~J}$. Patterson and J. Imberger, "Unsteady natural convection in a rectangular cavity," J. Fluid Mech. 100, 65 (1980).

${ }^{5}$ G. D. V. Davis, "Natural convection of air in a square cavity: A bench mark numerical solution," Int. J. Numer. Methods Fluids 3, 249 (1983).

'P. L. Quere and T. A. D. Roquefort, "Computation of natural convection in two-dimensional cavities with Chebyschev polynomials," J. Comput. Phys. 57, 210 (1985).

${ }^{7}$ T.-M. Wang and S. A. Korpela, "Convection rolls in a shallow cavity heated from a side," Phys. Fluids A 1, 947 (1989).

${ }^{8} \mathrm{H}$. P. Kuo and S. A. Korpela, "Stability and finite amplitude natural convection in a shallow cavity with insulated top and bottom and heated from a side," Phys. Fluids 31, 23 (1988).

${ }^{9}$ B. Ramaswamy, T. C. Jue, and J. E. Akin, "Finite element analysis of oscillatory flow with heat transfer inside a square cavity," AIAA J. 30, 412 (1992).

${ }^{10} \mathrm{~J}$. O. Wilkes and S. W. Churchill, "The finite-difference calculation of natural convection in a rectangular enclosure," AIChE J. 12, 161 (1966)

${ }^{11}$ T. C. Jue and B. Ramaswamy, "Natural convection with thermocapillary and gravity modulation effects in low-gravity environments," J. Spacecr. Rockets 29, 856 (1992)

${ }^{12} \mathrm{~J}$. Alexander, "Low-gravity experiment sensitivity to residual acceleration: a review," Microgravity Scí. Technol. 3, 52 (1990).

${ }^{13}$ J. Alexander, S. Amiroudine, J. Ouazzani, and F. Rosenberger, "Analysis
} 
of the low gravity tolerance of Bridgman-Stockbarger crystal growth II. Transient and periodic accelerations," J. Cryst. Growth 113, 21 (1991).

${ }^{14} \mathrm{~S}$. Biringen and G. Danabasoglu, "Computation of convective flow with gravity modulation in rectangular cavities," J. Thermophys. 4, 357 (1990).

${ }^{15}$ Y. Kamotani, A. Prasad, and S. Ostrach, "Thermal convection in an enclosure due to vibrations aboard a spacecraft," AIAA J. 19, 511 (1981).

${ }^{16} \mathrm{D}$. Jacqmin, "Stability of an oscillated fluid with a uniform density gradient," J. Fluid Mech. 219, 449 (1990).
${ }^{17}$ A. Farooq and G. M. Homsy, "Streaming flows due to $g$-jitter-induced natural convection," J. Fluid Mech. 271, 351 (1994).

${ }^{18} \mathrm{P}$. Roache, Computational Fluid Dynamics (Hermosa, Albuquerque, NM, 1972).

${ }^{19} \mathrm{~W}$. Zhang, J. Casademunt, and J. Viñals, "Study of the parametric oscillator driven by narrow band noise to model the response of a fluid surface to time-dependent accelerations," Phys. Fluids A 5, 3147 (1993).

${ }^{20}$ W. H. Press, B. P. Flannery, S. A. Teukolsky, and W. T. Vetterling, $\mathrm{Nu}$ merical Recipes (Cambridge University Press, Cambridge, 1986). 